\title{
Rôle et exploitation des barrages-réservoirs en cours de réalisation dans le bassin de la Seine, en vue de lutter contre les inondations et d'augmenter les débits d'étiage
}

\author{
Purpose and operation \\ of storage dams now under construction \\ in the Seine basin for flood control, \\ and to increase low-water discharge
}

PAR R. COULOMB,

INGÉNIEUR EN GHEF DU SERVIGE DES BARRAGES-RËSERYOIRS A LA DIRECTION TECHNIQUE DU PORT DE PARIS (PRÉFEcTURE DE LA SEINE), PARIS

\begin{abstract}
Difficultés concernant la réalisation et l'exploitation de barrages-réservoirs à buts multiples. Rappels sur les ouvrages déjáa en service. Conception des réservoirs dont la réalisation a été décidée: réservoir «Seine», près de Troyes, actuellement en construction (capacité: 205 millions de $\mathrm{m}^{3}$ ) et réservoir «Marne» près de Saint-Dizier (capacité : 350 millions de $\mathrm{m}^{s}$ ). Consignes d'exploitation de ces réservoirs. Influence du réservoir «Marne» sur le régime de la Marne et de la Seine (cas de la crue de janvier 1955).
\end{abstract}

Le but de cet exposé est de présenter, après un bref historique, les deux importants barrages-réservoirs que le département de la Seine a décidé de réaliser avec l'aide de l'Etat, afin d'abaisser d'une façon décisive la hauteur des grandes crues à Paris et de relever les débits d'étiage de la Seine et de la Marne, pour diminuer la pollution de ces rivières et faire face aux besoins en eau croissants de la région parisienne.

$$
\text { :**:* }
$$

La Seine roule à Paris, lors d'une crue comme celle de 1910 , plus de $2300 \mathrm{~m}^{3} / \mathrm{s}$ et déborde pardessus les parapets de la capitale après avoir

\begin{abstract}
Construction and operational difficulties associated with multi-purpose storage dams. Review of structures already in service. Design features of reservoirs to be built: "Seine" reservoir near Troyes (205 million cu.m-now under construction), and "Marne" reservoir near Saint-Dizier (350 million cu.m). Operating methods. How the "Marne" reservoir may be expected to affect conditions in the rivers Marne and Seine, considering the case of the January 1955 flood.
\end{abstract}

inondé de nombreuses agglomérations de banlieue. En été, c'est parfois un égoût aux eaux presque stagnantes, le minimum absolu du débit naturel $\left(35 \mathrm{~m}^{3} / \mathrm{s}\right.$ d'après les études de Belgrand et du Service de la Navigation de la Seine, répartis approximativement en $23 \mathrm{~m}^{3} / \mathrm{s}$ pour la Seine et $12 \mathrm{~m}^{3} / \mathrm{s}$ pour la Marne) étant du même ordre que la somme des débits pompés dans la rivière.

Aussi, l'idée de construire des barrages-réservoirs dans le bassin de la Seine dans le double but d'une part, de fournir de l'eau en été à diverses catégories d'usagers grâce à un renforcement du débit de la rivière, et d'autre part de lutter contre les inondations, remonte-t-elle à plus d'un siècle. 
C'est le deuxième de ces objectifs qui a été mis en avant après les crues de 1910 et de 1924 et c'est pour établir un programme de lutte contre les inondations qu'une Commission a été créée en 1925. Mais cette même Commission n'a retenu la nécessité de construire des barragesréservoirs que dans la mesure oì on leur trouverait d'autres utilisations susceptibles de gager en tout ou en partie les dépenses de construction. Et c'est plutôt pour atteindre le premier objectif (relèvement du débit d'étiage), qu'a été construite entre 1931 et 1949 la première série de barrages-réservoirs, dont nous allons parler; c'est aussi en raison du manque d'eau en été, que l'on est aujourd'hui si pressé de mettre en service la deuxième série de réservoirs, objet essentiel de notre propos.

Or, ces deux objectifs, écrêter les crues, relever les étiages, sont difficilement conciliables, même si l'on ne cherche pas, ce qui serait d'ailleurs sans grand intérêt, à régulariser le débit de la Seine autour de sa valeur moyenne, qui est de $275 \mathrm{~m}^{3} / \mathrm{s}$ à Paris. Si l'on ne dispose que d'un réservoir ayant une capacité du même ordre que le volume à prélever pour écrêter une seule crue importante de la rivière, il est même impossible de les poursuivre à la fois d'une façon convenable : en effet, si le réservoir est surtout destiné à lutter contre les crues, on le maintiendra vide, dans l'attente d'une crue dangereuse, qui le remplira pratiquement complètement, puis sans même attendre la fin de la crue, on commencera à le vider de crainte qu'une nouvelle crue n'arrive: on ne pourra donc songer à remplir le réservoir qu'en mars, une fois passée la période des grandes crues et alors, vu les débits généralement faibles de mars à juin, le réservoir sera presque vide au $1^{\text {er }}$ juillet, au moment oú il sera utile de renforcer le débit des rivières. Si, au contraire, on donne la priorité au renforcement des débits d'étiage, on remplira au plus tôt le réservoir, en écrêtant peut-être les petites crues, mais en courant le risque de le voir plein lors du passage d'une crue vraiment dangereuse.

La seule solution, si on désire régulariser d'une façon sensible la rivière, consiste à construire des réservoirs de grande capacité dont la partie supérieure restera vide durant la mauvaise saison pour n'être remplie qu'en cas de grande crue et dont la partie inférieure, remplie progressivement dès l'automne et le début de l'hiver, assurerait en tout état de cause une amélioration notable de l'étiage suivant.

Nous préciserons ces notions en définissant des consignes d'exploitation pour les deux grands réservoirs en cours de réalisation.

Ajoutons qu'en construisant les barrages-réservoirs, on a cherché également à utiliser les chutes ainsi créées pour produire de l'énergie hydro-électrique. Mais poursuivre ce troisième objectif en cherchant la production optimale d'énergie, est incompatible avec les deux autres. M. Pfahl, ingénieur en chef des Ponts et Chaussées, chef de la quatrième Circonscription ćlectrique à Limoges [1], a montré récemment à propos du bassin, très différent, à dire vrai, de la Dordogne, que, même avec une série de grands réservoirs dont celui de Bort-les-Orgues, l'exploitation des chutes dans un but énergétique avait entrainé en général l'ćcrêtement des petites crues, mais une fois sur deux seulement celui des crues les plus dangereuses, la recherche d'une grande hauteur de chute étant incompatible avec le maintien d'une tranche de crue importante. Si l'on ajoute qu'effectuer la vidange des réservoirs en été pour renforcer les débits d'étiage, comme c'est le cas dans le bassin de la Seine entraîne une production d'énergie à un moment où elle ne vaut presque rien, on se rend compte qu'il est impossible de poursuivre à la fois d'une façon satisfaisante trois buts si différents.

Aussi l'électricité a-t-elle toujours été considérée, dans le bassin de la Seine, comme un sous-produit assurant le financement des groupes et du bâtiment de l'usine, mais ne permettant pas de faire participer d'une facon importante les anciens concessionnaires, ou maintenant Electricité de France, à la réalisation du barrage-réservoir proprement dit. Seul, parmi les ouvrages dont nous allons parler, le petit barrage du Crescent sur la Cure est exploité dans un but vraiment hydro-électrique, la réserve ayant été dans ce cas partagée en deux, et le Département de la Seine contrôlant la tranche supérieure destinée à la lulte contre les crues et au renforcement des éliages. Dans les autres ouvrages, existants ou a comstruire, comporlant tous, sauf celui de Champaubert-auxBois, une usine électrique, le concessionnaire de celle-ci, Electricité de France, s'astreint à respecter la consigne d'exploitation fixée par le Département de la Seine, même s'il en résulte pour elle des inconvénients, quilte à les atlénuer dans le cas du barrage de PannesicreChaumard par la construction d'un barrage de compensation.

Ces généralités nous ont paru nécessaires pour siluer le cadre dans lequel s'est poursuivi le travail des ingénieurs de la Préfecture de la Seine depuis la décision du 14 janvier 1926 du ministre des Travaux Publics approuvant les conclusions de la Commission de 1925 déjà citée. Signalons de plus, que créer des réservoirs stockani les eaux d'hiver pour les restituer seulement l'été suivant, exigeaient que ceux-ci sojent implantés sur des terrains imperméables, alors que la seule lutle contre les inondations n'implique pas cette condition. Or, ceci limite 


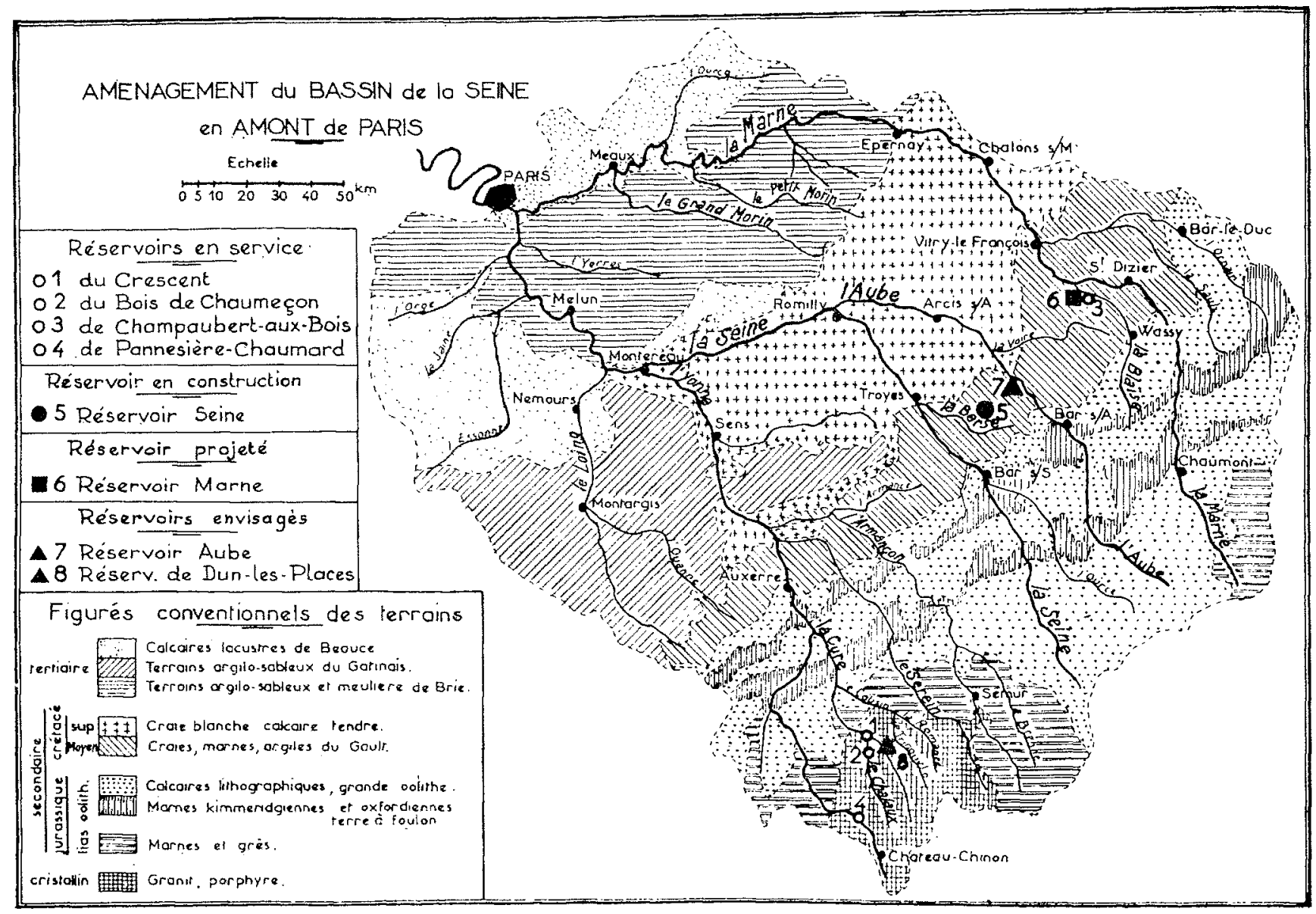

F1G. 1

Carte hydrogéologique du bassin de la Seine en amont de Paris.

beaucoup les possibilités d'établissement de barrages dans le bassin de la Seine, à l'amont de Paris.

La figure 1 donne la carte géologique de ce bassin et le tracé des principales rivières. Parmi les zones imperméables, nous devons écarter les terrains du lias situés tout à fait à l'amont du bassin, en des points où les débits des rivières sont encore très faibles, et les argiles et marnes des plateaux de la Brie et du Gâtinais, sillonnés seulement par des ruisseaux et dominant les vallées des rivières importantes, ce qui empêche d'y dériver leurs eaux. Les deux seules régions propices à l'établissement de réservoirs sont donc celles des granites du Morvan et celles des argiles du Gault de la Champagne humide. Tous les barrages retenus par la Commission de 1925 se trouvent d'ailleurs dans une de ces deux zones.

$\mathrm{Si}$, dans le Morvan, les sites géologiques possibles pour la construction de barrages sont nombreux, ils ne se prètent qu'à l'établissement de retenues relativement petites sur des rivières à faibles débits. L'affleurement des argiles du Gault, de $10 \mathrm{~km}$ de largeur au sud, $35 \mathrm{~km}$ de largeur au nord, recoupe par contre des rivières importantes (Serein, Seine, Aube, Marne) dans des zones où leurs débits sont déjà importants.

Néanmoins, les quatre ouvrages qui ont été réalisés en première urgence et sont en service depuis de longues années sont situés dans le Morvan, à l'exception d'un petit réservoir dans la Champagne humide, qui n'utilise pas toutes les possibilités du site.

Ce sont (cf. fig. 1) :

\section{a) Dans Le Morvan :}

- le réservoir du Crescent, sur la Cure, de 14,6 millions de $\mathrm{m}^{3}$, dont une tranche de 4 millions de $\mathrm{m}^{3}$ est réservée au Département de la Seine.

- le réservoir du Bois de Chaumecon, sur le Chalaux (19 millions de $\mathrm{m}^{3}$ );

- le réservoir de Pannesière-Chaumard, sur l'Yonne (82,5 millions de $\left.\mathrm{m}^{3}\right)$, le seul important et le plus récent. 
b) Dans la Champagne humide, le réservoir de Champaubert-aux-Bois (23 millions de $\mathrm{m}^{3}$ ), en dérivation sur la Blaise.

Ce programme, achevé en 1949, représentait donc une accumulation de $130000000 \mathrm{~m}^{3}$ au total pour l'écrêtement des crues et le relèvement des débits d'étiage. Ce chiffre est notoirement insuffisant pour atténuer d'une façon sensible les inondations. En outre, si Pannesière a permis de renforcer notablement l'étiage de la Seine à l'amont de Paris, le petit réservoir de Champaubert, avec sa capacité réduite et ses lâchures limitées à $8 \mathrm{~m}^{3} / \mathrm{s}$ en pointe et $3 \mathrm{~m}^{3} / \mathrm{s}$ en moyenne, n'a pas résolu le grave problème posé par la faiblesse des débits d'étiage de la Marne.

Aussi, le Département de la Seine, dès l'achèvement du réservoir de Pannesière, poursuivit activement les études de barrages-réservoirs beaucoup plus vastes, dont les sites possibles étaient, à peu de choses près, déterminès depuis 1920, mais qui n'étaient envisagćs qu'en deuxième ligne par la Commission de 1925.

Compte tenu des divers aspects de la question (prix et incidences sociales notamment), le choix s'est porté sur la construction de deux ouvrages, le réservoir «Seine» et le réservoir «Marne» (cf. fig. 1) situés dans la Champagne humide, en dérivation, respectivement, sur la Seine et sur la Marne.

a) LE RÉSERvoIr «SEINe 》 (cf. fig. 2), situé à l'est de Troyes, est alimenté par un canal d'amenée de $13 \mathrm{~km}$ environ, capable de prélever jusqu'à $180 \mathrm{~m}^{3} / \mathrm{s}$ en Seine, soit plus de $50 \%$ du débit maximal connu à la prise d'eau. La retenue, d'une capacité de 205 millions de $\mathrm{m}^{3}$, s'étend sur 2300 ha environ. Elle est ceinturée par quatre groupes de digues en limon argileux compacté, dont la plus importante atteint $25 \mathrm{~m}$ de hauteur maximale. Les eaux emmagasinées seront restituées par un canal empruntant les vallées de la Morge et de la Barse et se divisant, à Ruvigny, en deux branches se jetant, l'une en Seine à l'amont de Troyes, l'autre dans la Vieille Seine à Baires. Ces canaux de restitution permettent d'effectuer des lâchures de $35 \mathrm{~m}^{3} / \mathrm{s}$.

L'emprise totale des ouvrages dépasse 2800 ha. Le coût de l'opération, déclarée d'utilité publique par décret du 25 septembre 1959, est estimé à 105 millions de $F$ auxquels s'ajoute 1 million de $F$ pour la reconstitution du potentiel agricole et forestier détruit par le réservoir. Les principaux ouvrages sont actuellement en construction et l'achèvement du réservoir est prévu pour 1965.

b) Le réseryoir 《Marne 》 (cf. fig. 3) se présente d'une facon analogue au réservoir «Seine». Il recouvre le réservoir actuel de

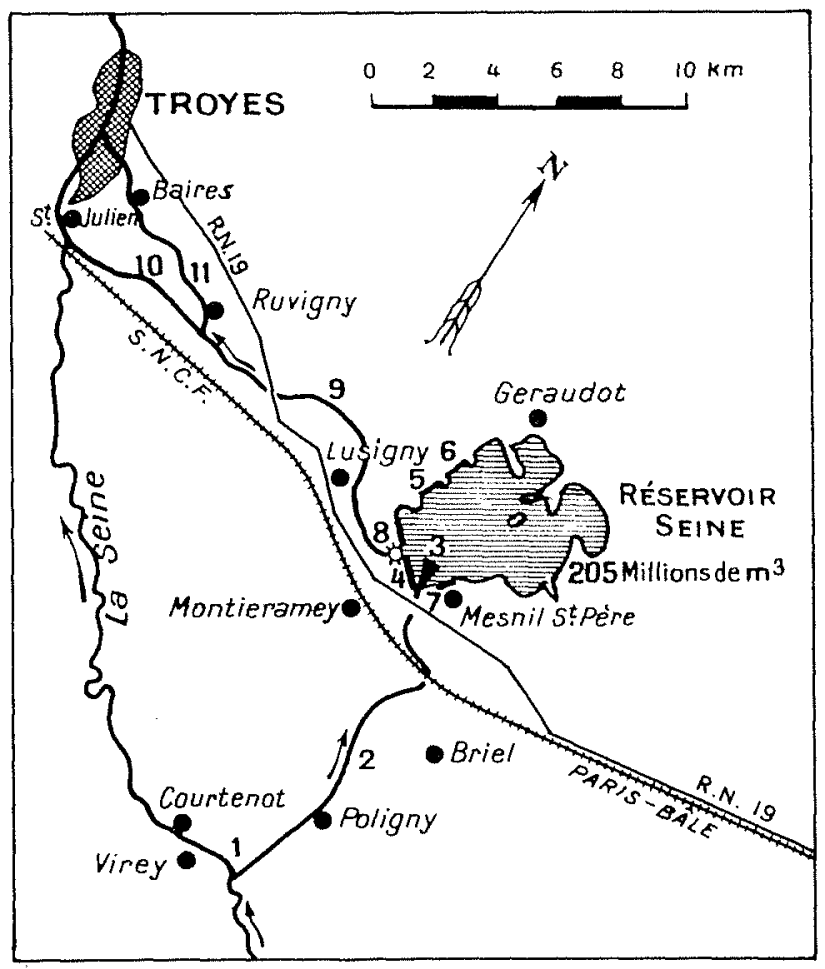

Fig. 2

Plan du réservoir \& Seine»

1: Prise d'eau. - 2 : Canal d'amenée. - 3: Déversoir d'extrémité. - 4 : Digues de la Morge et de Beaumont. 5 : Digue de Chavaudon. - 6: Digue de Géraudot. - 7 : Digue de Mesnil-Saint-Père. - 8: Usine de la Morge (E.D.F.). - 9 : Canal de restitution de la Morge. - 10): Canal de restitution de Saint-Julien. - 11: Canal de restitution de Baires.

Champaubert-aux-Bois, dont la capacité de la rigole d'amenée est portée de 15 à $33 \mathrm{~m}^{3} / \mathrm{s}$ et dont le plan d'eau est relevé de 139,00 N'.G.F. à 139,70 N.G.F. La cuvelte actuelle est complétée par des endiguements en limon argileux compacté de faible hauleur, la digue principale de Giffaumont, au sud, alteignant $19,20 \mathrm{~m}$ de hanleur maximale. Le canal d'amenée au réservoir "Marne», alimenté grâce à un barrage en rivière situé dans la partie amont de Saint-Dizier, a une capacité de $360 \mathrm{~m}^{3} / \mathrm{s}$ et se déverse dans l'actuel réservoir de Champaubert. La restitution en Marne est assurée par un canal de courte longueur, permettant d'effectuer des lâchures de $50 \mathrm{~m}^{3} / \mathrm{s}$.

L'emprise totale des ouvrages, y compris celle du réservoir de Champaubert, dépassera 5000 ha. Le plan d'eau lui-même aura une surface de 4700 ha et recouvrira les trois petils villages de Champaubert-aux-Bois, Nuisement-aux-Bois, et Chantecoq. Le coût de l'opération est estimé à 120 millions de $F$. Seules des acquisitions amiables d'immeubles et de terrains ont, à ee jour, été effectuées, l'enquête d'utilité publique n'étant 
pas encore ouverte. En raison de ses incidences sociales, la réalisation de cet ouvrage n'a pu en effet être décidée que tout récemment.

\section{Exploitation du réservoir “Marne» (1) :}

Le réservoir «Seine» joue par rapport à la haute Seine un ròle de régularisation en tous points analogue à celui du réservoir "Marne * par rapport à la Marne. Aussi, nous limiteronsnous désormais au seul cas du réservoir «Marne », pour la clarté de l'exposé. C'est d'ailleurs le plus important des deux, en raison non seulement du meilleur site du réservoir, mais aussi parce que la Marne à Saint-Dizier est une rivière plus importante que la Seine à Troyes.

Le débit maximal possible du canal d'amenée du réservoir «Marne », $360 \mathrm{~m}^{3} / \mathrm{s}$, permet de ramener les crues exceptionnelles à Saint-Dizier au niveau des crues annuelles et de supprimer ces dernières. Il permet d'assurer une protection pratiquement complète de Vitry-le-François ef de Châlons-sur-Marne, et d'abaisser, comme nous le verrons, le niveau des grandes crues à Paris de $70 \mathrm{~cm}$. Mais, pour obtenir ce résultat, il fant pouvoir emmagasiner dans le réservoir un volume d'eau qui, pour les grandes crues, est de l'ordre de 150 millions de $\mathrm{m}^{3}$. La capacité du réservoir (350 millions de $\mathrm{m}^{3}$ ) permet de réserver cette tranche de crue jusqu'à fin février (sauf pendant les derniers jours du mois, si aucune crue n'est annoncée), le surplus du réservoir (200 millions de $\mathrm{m}^{3}$ ) étant rcmpli lui-même essentiellement par les hautes eaux et les crues de novembre à février.

Compte tenu d'une tranche morte de 10 millions de $\mathrm{m}^{3}$, destinée à préserver le poisson, on dispose, si le réservoir est plein, de 340 millions de $\mathrm{m}^{3}$ pouvant être vidangés du $1^{\mathrm{er}}$ juillet au 31 octobre à une cadence maximale de $50 \mathrm{~m}^{3} / \mathrm{s}$ et moyenne de $32 \mathrm{~m}^{3} / \mathrm{s}$, ce qui permettra, associé avec le réservoir «Seine » et les réservoirs existants, de maintenir le débit naturel (avant pompages) à Paris nettement au-dessus de $100 \mathrm{~m}^{3} / \mathrm{s}$ chiffre suffisant, mais éminemment souhaitable, compte tenu des nouveaux pompages envisagés dans les 10 ans à venir.

La capacité du réservoir est donc suffisante, pourvu gue l'on puisse effectivement lc remplir. Or, l'application sur 25 campagnes de remplissage (du $1^{\text {er }}$ novembre au 30 juin) de la consigne que nous allons résumer, montre que le réservoir aurait toujours pu être rempli à plus de

(1) Le lecteur trouvera plus de détails sur celte question dans un article récent $[2]$.

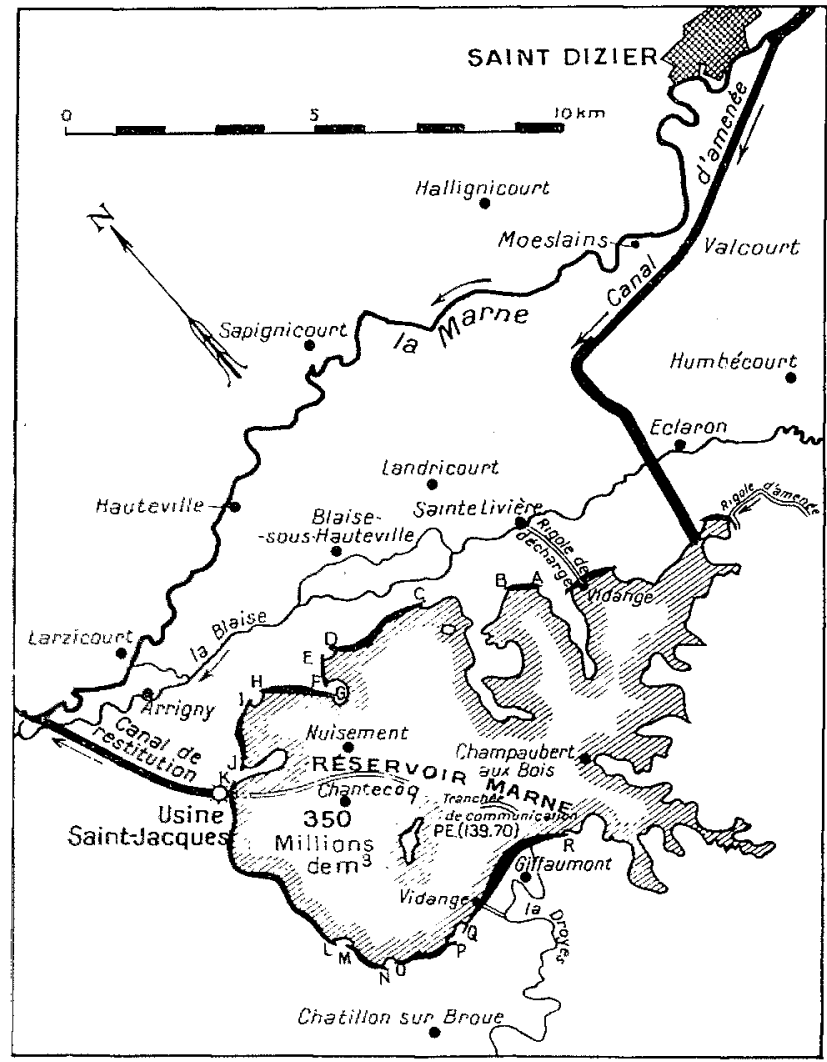

FIG. 3

Plan du réservoir «Marne».

$A B, C D, \ldots O P, Q R$, digues à construire.

320 millions de $\mathrm{m}^{3}$ (sauf les années extrêmement sèches telles que 1920-1921 et 1953-1954) et que plus de la moitié des années, il aurait été plein au $1^{\text {er }}$ juillet, à 10 millions de $\mathrm{m}^{3}$ près.

Cette consigne, satisfaisante par conséquent pour le remplissage du réservoir, est pourtant trẻs prudente pour les crues.

D'une façon schématique, l'année se divise en deux périodes :

- remplissage du $1^{\text {er }}$ novembre d'une année au 30 juin de l'année suivante, et

- vidange du $1^{\text {er }}$ juillet au 31 octobre.

Pour la première période, la plus délicate, on pourrait concevoir la consigne de la facon suivante : remplir à partir du $1^{\text {re }}$ novembre avec tout le débit disponible jusqu'à 200 millions de $\mathrm{m}^{3}$, de facon à s'assurer cette tranche d'étiage pour l'été suivant, et réserver la tranche de crue de 150 millions de $\mathrm{m}^{3}$ jusqu'à la fin de février pour absorber les crues, chaque remplissage lors d'une crue étant suivi d'une vidange pour ramener le volume à 200 millions de $\mathrm{m}^{3}$ en prévision de crues ultérieures.

Mais cette consigne est trop simple et en fait dangereuse. La tranche de 150 millions de 
$\mathrm{m}^{3}$ est juste suffisante pour absorber une très grande crue. Mais si la tranche d'étiage est déjà pleine, il en résulte un remplissage complet du réservoir, qui nécessite une vidange postérieure importante, difficile à faire admettre, car elle prolonge la crue, et longue à réaliser. Si une deuxième crue suit de près la première, le réservoir ne peut l'absorber complètement.

$\mathrm{Si}$, au contraire, on remplit progressivement et prudemment la tranche d'étiage de 200 millions de $\mathrm{m}^{3}$, on peut emmagasiner une partie de la première crue qui survient dans cette tranche d'étiage; le volume à vidanger se trouve diminué d'autant et l'on est prêt plus tôt pour faire face à une nouvelle crue.

Aussi, après quelques tâtonnements, est-on arrivé à la consigne schématisée par la figure 4 (graphique d'exploitation), qui prévoit un remplissage par paliers successifs de la «tranche d'étiage » (200 millions de $\mathrm{m}^{3}$ ) jusqu'au 15 février, puis, si aucune crue n'est annoncée, le

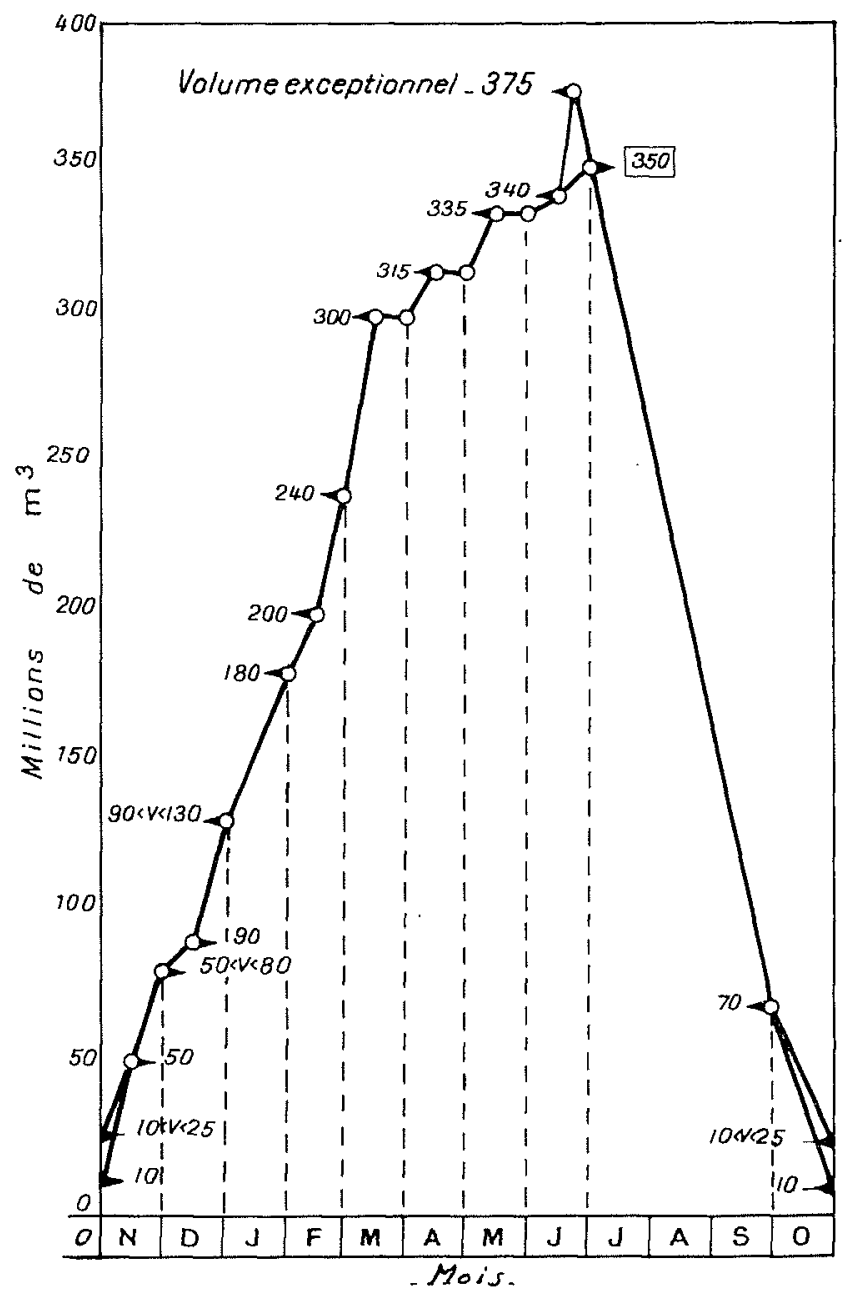

Fig. 4

Graphique d'exploitation du réservoir "Marne». remplissage de la tranche de crue, effectué également par paliers de façon à pouvoir écrêter des crues de printemps ou d'été, moins importantes, mais préjudiciables à l'agriculture de la vallée.

La consigne pendant la période de vidange est plus simple, car on a renoncé à moduler exactement les lâchures en fonction des besoins à Paris; vu l'éloignement du réservoir, le rôle des pompages riverains et celui de la nappe, cette modulation aurait d'ailleurs ćté illusoire. En cas de grande sécheresse, on augmentera simplement les lâchures prévues à $35 \mathrm{~m}^{3} / \mathrm{s}$ de juillet à septembre pour les porter à $50 \mathrm{~m}^{3} / \mathrm{s}$, débit que l'on maintiendra pendant des semaines, pour être sûr d'en retrouver l'essentiel à Paris. En octobre, la cadence de la vidange dépendra de la sécheresse du mois; si le mois est humide, on a en effet intérêt à garder un peu d'eau pour l'année suivante et à maintenir une certaine hauteur de chute pour le fonctionnement de l'usine électrique.

Pour contrôler l'efficacité de cette consigne et de celle, très analogue, du réservoir «Seine» dans des conditions aussi voisines que possible de l'exploitation future, nous exploitons jour par jour les deux réservoirs comme s'ils existaient, connaissant les débits aux prises d'eau et à Paris, les renseignements méléorologiques et ceux du service d'annonce des crues.

$$
\text { *** }
$$

Influence du réservoir "Marne" sur les crues de la Marne et de la Seine :

Après avoir montré comment exploiter un amćnagement à double fin, dont nous avons donné les caractéristiques, nous voudrions montrer son influence réelle dans la réduction de l'importance des crues à Paris, et ce, malgré son éloignement de la capitale $(350 \mathrm{~km})$ et la présence entre Saint-Dizier et Paris de vastes champs d'inondation qui atténuent la erue et que l'on utilisera moins bien lors du fonctionnement du réservoir (toute diminulion du niveau de la crue entraînant une diminution du volume de la retenue naturelle que constiluent les champs d'inondation).

L'étude de cetle influence a pu récemment être eflectuée en examinant le rôle qu'aurait joué le réservoir «Marne», s’il avait existé, lors des deux grandes crues bien connues de 1910 et 1955, grâce au Service Central hydrologique du Ministère des Travaux Publics, et notamment à M. Lallement, en utilisant la méthode qu'emploie ce service pour l'annonce des crues. 
Cette méthode, due à M. Bachet, ingénieur général des Ponts et Chaussées [3], et utilisant des réglettes de correspondances, permet, connaissant les hauteurs d'eau en un point de la rivière, de déterminer celles que nous aurons ultérieurement en d'autres points situés à l'aval. Ces dernières sont le résultat de deux effets : une propagation de l'onde de crue et une atténuation dépendant des caractéristiques de l'onde et qui est due aux conditions particulières du lit de la rivière et des champs d'inondation.

L'emploi de la méthode implique l'existence de stations de mesures de hauteurs et de débits le long de la Marne et de ses affluents et sur la Seine à l'amont immédiat de son confluent avec la Marne.

Fort heureusement, les réglettes existantes permettaient d'effectuer convenablement ce travail. Tout d'abord, l'écrêtement des crues à Saint-Dizier, à l'aval immédiat de la prise d'eau, résulte simplement de la considération de la courbe hauteurs-débits, compte tenu des débits prélevés dans le réservoir. La réglette SaintDizier-Vitry-en-Perthois (sur la Saulx, affluent de la Marne)-Damery (sur la Marne à $11 \mathrm{~km}$ à l'aval d'Epernay) permet d'obtenir l'onde de crue à Damery, d'une part sans le réservoir «Marne» et d'autre part en présence de celui-ci (cf. fig. 5 pour le cas de la crue de janvier 1955; on contate que l'abaissement est de $1,60 \mathrm{~m}$ à Saint-Dizier et seulement de $0,60 \mathrm{~m}$ à Damery, où existe un vaste champ d'inondation). La coïncidence de l'onde de crue réelle enregistrée à Damery en 1955 avec celle donnée par la réglette (en l'absence du réservoir) a permis de constater la précision de la méthode, non seulement pour obtenir le maximum de la crue, but essentiel de l'annonce des crues, mais aussi pour obtenir la forme de l'onde de crue. Aussi peut-on, avec une certaine confiance, utiliser les

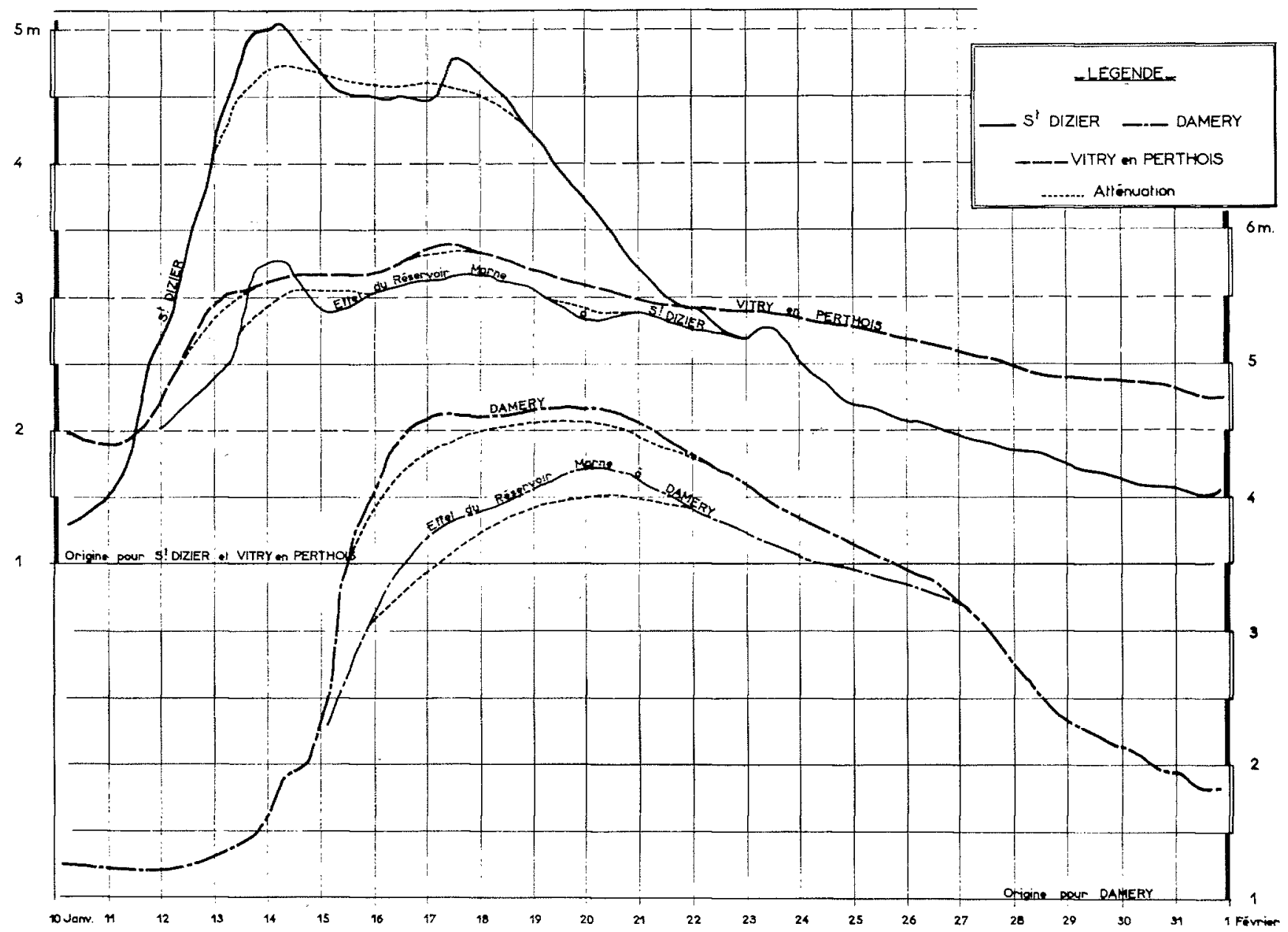

Fig. 5

Méthode Bachet.

Effet du réservoir «Marne» pendant la crue de janvier 1955 à Saint-Dizier et Damery. 


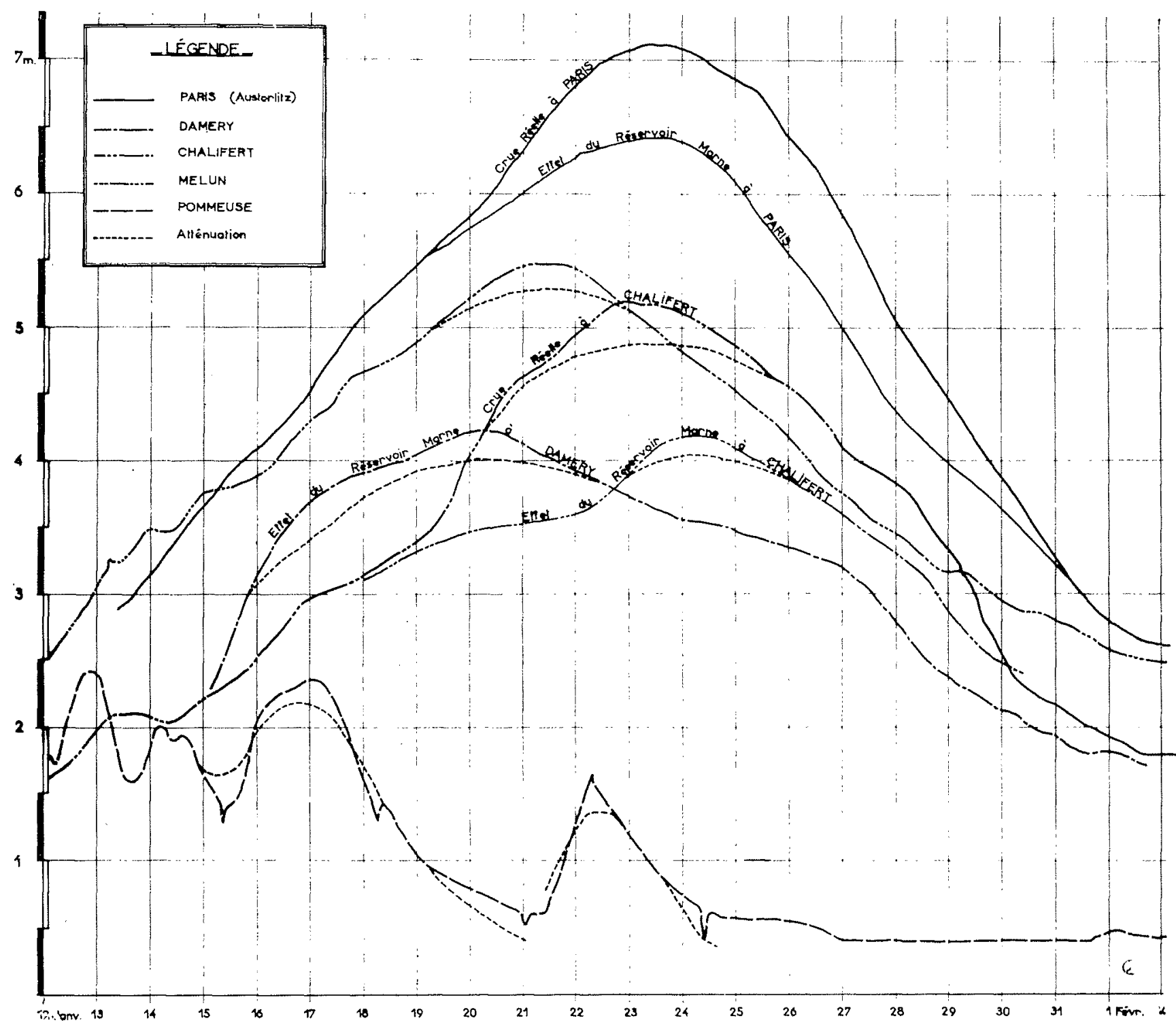

FIG. 6

Méthode Bachet.

Effet du réservoir «Marne» pendant la crue de janvier 1955 à Chalifert et Paris.

deux réglettes suivantes : Damery - Pommeuse - Chalifert (2) et Chalifert-Melun (3) Paris (Austerlitz) pour étudier la propagation de la crue modifiée par le réservoir «Marne» jusqu'à Chalifert, puis jusqu'à Paris (cf. fig. 6 pour le cas de la crue de janvier 1955).

On en a déduit qu’à Paris-Austerlitz l'abaisse-

(2) Pommeuse est situé sur le Grand Morin à l'aval immédiat de Coulommiers, et Chalifert sur la Marne, à $30 \mathrm{~km}$ à l'aval de Meaux.

(3) Sur la Seine à l'amont de Paris. ment du niveau des crues de 1910 et 1955 aurait été respectivement de $0,80 \mathrm{~m}$ et $0,70 \mathrm{~m}$. Si l'on tient compte du réservoir «Seine», les mêmes crues auraient été abaissćes respectivement de $1,20 \mathrm{~m}$ et $1,06 \mathrm{~m}$ (cf. fig. 7 pour le cas de la crue de janvier 1955).

C'est dire qu'à Paris, avec ces deux ouvrages, les crues comme celles de 1924 ou 1955 , qui se produisent en moyenne tous les 30 ans, n'entraîneront plus de débordements, el les crues centenaires, comme celle de 1910 , seront ramenées au niveau de crues se produisant actuellement tous les 20 ans, soit $6,90 \mathrm{~m}$ au pont d'Austerlitz. 


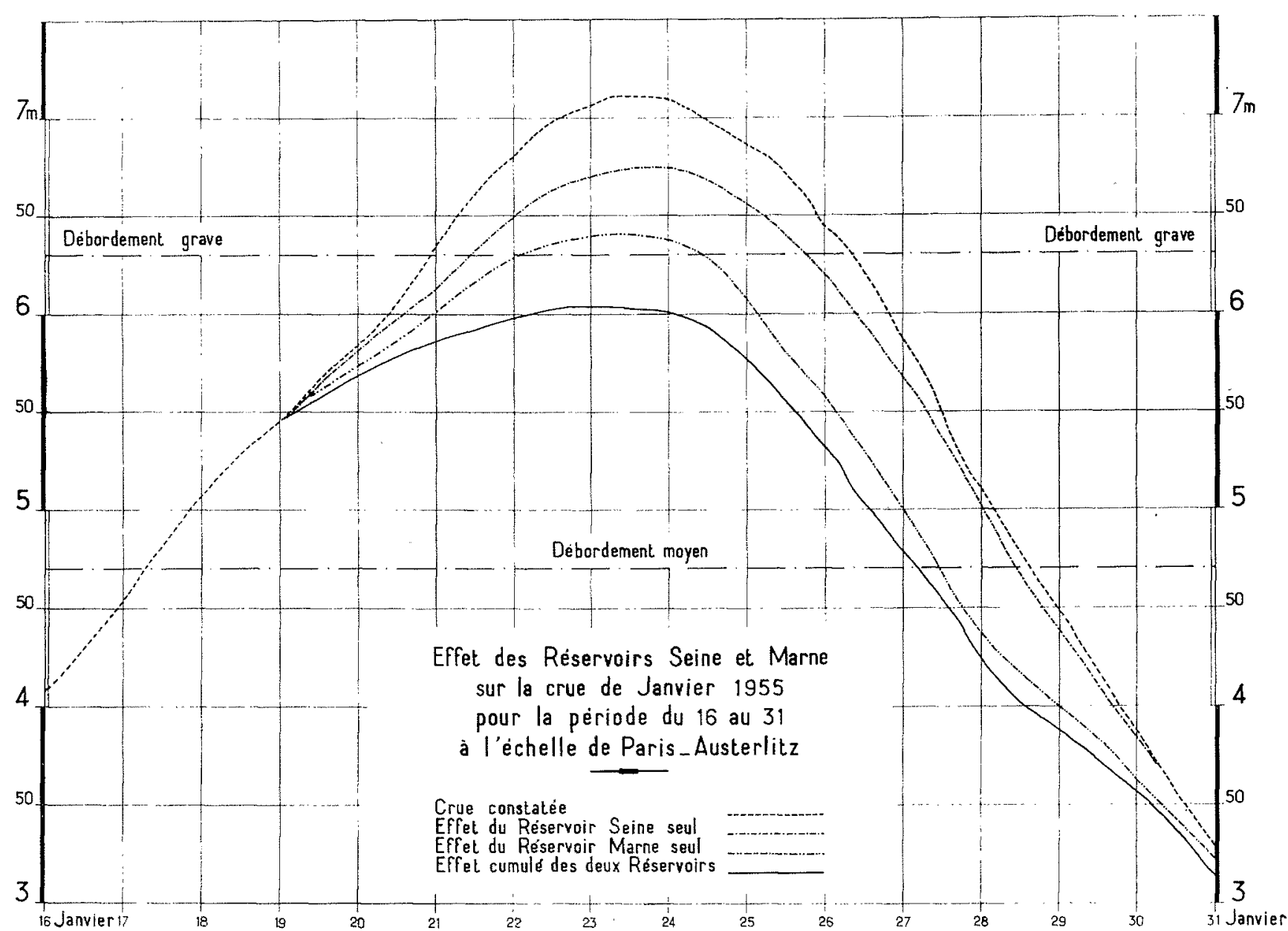

FiG. 7

Effet des réservoirs «Seine» et «Marne» en 1955 à Paris-Austerlitz.

\section{Références}

[1] PFaHL (H.). - L'exploitation des barrages conçus dans le but de la production d'énergie électrique; leur incidence sur les crues dans le cas du bassin de la Dordogne. Annales des Ponts et Chaussées, $133^{\circ}$ année, $n^{\circ} 3$, mai-juin 1963.

[2] Coulom i (R.). - L'aménagement du bassin de la Seine au moyen de barrages-réservoirs : écrêtement des crues, amélioration du débit d'étiage, conception des ouvrages et exploitation. Annales des Ponts et Chaussées, $133^{e}$ année, $n^{\circ} 4$, juilletaoût 1963.

[3] BACHET. - Note sur la propagation et l'annonce des crues. Annales des Ponts et Chaussées, mai-juin 1934.

\section{1. - COMPLËMENT ET CONCLUSION Président : M. Taluratu}

M. Couloms ajoute à sa communication la conclusion ci-après :

«Ces chiffres relatifs aux inondations doivent être associés à l'heureux effet des réservoirs sur le renforcement des débits d'étiage qui pourront être maintenus, avant pompages, au-dessus de $100 \mathrm{~m}^{3} / \mathrm{s}$. Pour préciser le rôle des barrages-réservoirs dans ce domaine et relier cet exposé à ceux d'hier (4), on peut d'ailleurs indiquer d'après le rapport de 1962 du Groupe d'études «Bassin de la Seine» de la Délégation générale au District de la Région de Paris, que même er tenant compte de l'augmentation envisagée des pompages d'ici 1973, les débits effectifs, grâce à ces deux barrages, et même dans le cas d'un étiage très bas associé à des pointes de pompage, seraient respectivement augmentés ainsi :

" - Seine, à l'amont du confluent avec la Marne, $22,5 \mathrm{~m}^{3} / \mathrm{s}$ au lieu de 17 actuellement;

«- Marne, $33,5 \mathrm{~m}^{\mathrm{s}} / \mathrm{s}$ au lieu de 2,5 ;

" - Seine après le confluent, $54 \mathrm{~m}^{3} / \mathrm{s}$ au lieu de 18 .

«Encore ces chiffres ne prennent-ils en compte que les débits moyens de vidange des réservoirs (soit $20 \mathrm{~m}^{3} / \mathrm{s}$ pour le réservoir «Seine» et $35 \mathrm{~m}^{3} / \mathrm{s}$ pour le réservoir «Marne ») au lieu des débits pour lesquels seront construits les canaux de restitution $\left(35 \mathrm{~m}^{3} / \mathrm{s}\right.$ pour le réservoir «Seine» et $50 \mathrm{~m}^{3} / \mathrm{s}$ pour le réservoir «Marne»).

"Il en résulte que si les effluents polluant la rivière ne s'accroissent pas d'ici à 1973 , et si j'ai bien noté hier les indications de M. Coutris, la Marne, actuellement de catégorie 3 entre Meaux et Paris, passerait, du seul fait de la dilution, en catégorie 1 ; la Seine à Ivry serait sûrement en catégorie 2 , au lieu d'être à la limite

(4) « La Pollution des rivières », La Houille Blanche, n०6, 1963. 
de la catégorie 3 et, après son confluent avec la Marne, elle resterait en catégorie 2 avec un débit d'étiage triple du débit actuel.

«Certes, ceci n'enlève xien de leur intérêt aux autres mesures à prendre pour éviter la pollution, ne fût-ce que pour parer aux cas exceptionnels où le remplissage des réservoirs aurait été défectueux.

«L'ensemble de ces résultats montre nénumoins que le but de régularisation poursuivi par le Département de la Seine et l'Etat peut être atteint moyennant la dépense relativement réduite de 225 millions de francs actuels, par la seule création de deux barrages-réservoirs, fort importants certes, mais ne présentant pas de difficultés techniques dirimantes. Il est cependant probable, qu'ultérieurement, à la fin du programme duo-décennal 1964-1975, ou au début du programme suivant, de nouveaux barrages-réservoirs devront être construits sur l'Aube ou sur la Cure, où deux réserves, respectivement de 146 et 60 millions de $\mathrm{m}^{3}$ peuvent être créées.»

M. le Président remercie vivement M. Coulomb de cet exposé et ouvre la discussion.

\section{II. - DISCUSSION}

M. Michon trouve relativement faible le relèvement des débits d'étiage de Ja Seine de 17 à $22,5 \mathrm{~m}^{3} / \mathrm{s}$ du fait de la présence du barrage «Seine».

M. Couloms indique que le passage de 17 is $22,5 \mathrm{~m}^{3} / \mathrm{s}$ résulte du fait qu'il y a deux phénomènes qui font la course entre eux. D'une part, on renforce les débits de la rivière, d'autre part, on installe de nouvelles stations de pompage et on augmente les prélèvements des stations existantes. D'autre part, nous l'avons indiqué, ce calcul est pessimiste, car il correspond à un débit de restitution de $20 \mathrm{~m}^{3} / \mathrm{s}$, alors que le canal de restitution du réservoir "Seine» est concu pour $35 \mathrm{~m}^{3} / \mathrm{s}$.

M. Ferry souligne l'antinomie qu'a bien montrée M. Coulomis entre l'utilisation d'un réservoir pour limiter l'importance des crues et son utilisation pour améliorer les étiages. Il croit utile d'insister particulièrement sur l'incompatibilité entre l'usage d'un réservoir à des fins hydrauliques (réduction des crues ou améliorations des étiages d'été), et son utilisation énergétique. Lorsqu'on vise des fins multiples, on est conduit à attribuer une partie du volume du réservoir à chaque fonction; or, le coût d'une unité de volume emmagasinc ne décrô̂t pas avec la hauteur de l'ouvrage. Par contre, la valeur énergétique d'un mètre cube emmagasine décroît par suite de la perte de chute. Il serait done généralement plus économique, dans la mesure où les sites le permettent, de créer des ouvrages distincts pour chaque fonction, de telle manière qu'un réservoir à usage énergétique puisse être utilisé sur la totalité de sa hauteur.

M. Covlonz répond que c'est en partie rrai. Dans le seul cas où l'on ait fait un seul ouvrage séparé en deux tranches, celui du Crescent, le département de la Seine a profité du fait que la Société d'Etudes des Chutes de la Cure construisait cet ouvrage pour le faire surélever, à ses frais, de 4 mètres. Cette manière de faire a coûté moins cher que si le Département avait construit entièrement un barrage lui-même.

M. FERRY pense que cela ne doit être plus avantageux que dans des cas particuliers exceptionnels. Si la hauteur d'un barrage à fins énergétiques n'est pas poussée au maximum techniquement permis par le site, c'est généralement parce que le coût des submersions devient très élevé et renchérit à l'excès le prix du mètre cube supplémentaire emmagasiné. Il serait donc préférable de chercher un autre site pour l'ourrage a fins hydrauliques.

D'autre part, si l'on adjoint à un ouvrage à fins liydrauliques une usine productrice d'énergie, celle-ci dtant considérée comme un sous-produit, on obtient une energie dont la raleur se limite pratiquement à celle du charbon économisé dans les centrales thermiques marginales. Cela ne permet pas à l'énergie de renter autre chose que l'usine hydraulique, sans aucune participation au con̂t du barrage. Cette situation ne peut que s'aggraver avec la diminution des dépenses de combustibles par $\mathrm{kWh}$ dans les centrales modernes et le déclassement des unités vétustes à forte consommation.

M. le Président remercie M. Fenny de ces précisions yui ont permis de sérier les problèmes et de montrer que des solutions différentes pourraient etre adoptées. Les ingénieurs pensent a ces solutions, mais le point sur lequel ils buttent est l'obtention des credits.

M. le Président n'est pas tout à fait convaincu par lo lait qu'en adoptant cette solution on arriverait à faire des économies.

M. Jamse ajoute, au sujet de l'écrêtement des erues, qu'il y a déjà eu des travaux, en particulier ceux des services de Navigation de la Seine, qui ont permis d'obtenir un effet de $0,70 \mathrm{~m}$ sur l'écrètement des crnes.

Cet effet va s'ajouter à celui qui est prévu $(1,20 \mathrm{~m}$ pour des crues importantes, grâce anx nouveaux ouvrages de «Seine» et de «Marne»).

M. Covlonb dit que l'abaissement est en effet, de $1,20 \mathrm{~m}$ par rapport in $\mathrm{la}$ crue de 1910 , corrige en tenant compte de la suppression de l'écluse du bras de la Monnaie par les Services de la Navigation en 1926.

M. Michon demande la date probable de mise en service du réservoir «Marne».

M. Coulomb ne peut répondre car l'enquête d'utilité publique n'est pas encore ouverte. Lille s'ouvrira sans doute vers la fin de l'année. Il $y$ a trois villages a exproprier et des oppositions importantes sur le plan public, mais tout le monde est pressé de voir sortir cet ouvrage. Les choses devaient done aller relativement vite.

La Délégation Générale au District de la kégion de Paris désire que ce harrage-réservoir soit terminé pour 1970. Il semble impossible de faire mieux.

M. ANDnÉ demande si l'on ne pourrait pas continuer à calculer les débits naturels de la Seine, malgré toutes ces influences, en particulier, pour les étiages qui apparaitront comme des différences faibles entre volumes importants, et ceci en vue de poursuivre les séries stalistiques déjà longues des débils de la Seine.

M. Coulomis pense que cela sera très difficile; les dernières statistiques sur les débits naturels d'étinge de la Seine remontent à des études de Belgrand, consacrées à la péliode de 1850-1865, époque où il n'y avait pas encore de barrage à Paris.

Depuis lors, on a barré la rivière, on prend de l'eau en de nombreux endroits et on la rejette ailleurs avec un certain décalage dans le temps par rapport au moment où l'on effectue les prélèvements.

A l'heure actuelle, ces débits sont mal connus et ne peuvent être évalués qu'en tenant compte de renseignements très imprécis (5).

M. Covlomi ne s'occupe pas directement de ces questions et ne peut donner d'autres précisions.

M. le président remercie à nouvean M. Couloms et les personnes ayant pris part à la discussion.

5) Voir : B. Gaspand. - Note sur la détermination des débits de la Seine à Paris. Annuatre II 1951, S.H.F., Jaris. 